above the bottom. In Patella, where secondary gills have appeared, the osphradia are vestigial because material is not normally carried into the reduced mantle cavity, while in the Opisthobranchs, Scaphander and Aplysia, it is reduced probably in correlation with rejection of sediment by cilia on the edge of grooves on the parapodia which conduct the water currents into the mantle cavity.

It is therefore suggested that the sense cells in the osphradium form a tactile organ which responds to contact stimuli, the impulse being conveyed by way of the osphradial nerve to the visceral ganglia. Blocking of the mantle cavity with sediment is a supreme danger to Gastropoda, and the importance of such a receptor is clear. The invariable association between ctenidum and osphradium is explained on this view, because the former creates the current, in which the quantity of sediment is estimated by the latter. The universal presence of the osphradium, irrespective of the habitat and of the food, is also explained. It is hoped later to follow up this biological study with an experimental analysis of the receptive capacities of the osphradium.

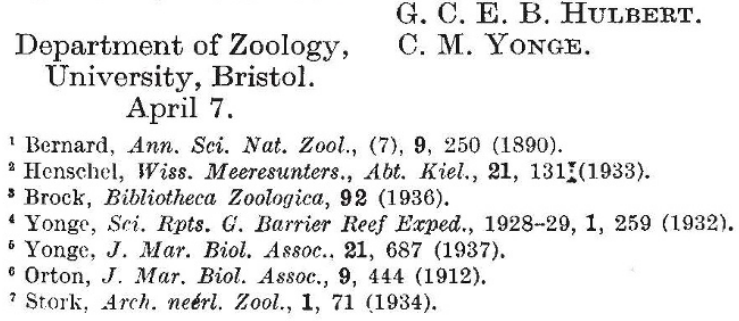

\section{Lunar Periodicity in Homo sapiens L.}

THE belief in a connexion between sexual periodicity and the moon is very ancient ${ }^{x}$. In particular, it is commonly thought that there is some connexion between the moon and menstruation. De Lee ${ }^{2}$ stated, without giving any evidence, that the majority of women menstruate during the new moon, thus associating the periodicity with the synodical lunar month of $29 \cdot 55$ days. Arrhenius $^{3}$ concluded from data of nearly 12,000 menstruations that there is a slight tropical lunar rhythm of $27 \cdot 32$ days, and that the average duration of the menstrual cycle is just under 27 days.

We have reinvestigated these questions with data of more than 10,000 menstruations. We find a great variation in the duration of the cycle, both in one individual and between different individuals. The average duration in our cases during 1928-30 was 29 days, but there was a clear inverse correlation between age and the duration of the cycle. Thus the cycle became shorter by a day with every five or six years increase in age, the oldest group (born before 1891) giving an average of 26.9 days, and the youngest (born after 1905) of 30.2 days. If, therefore, the average cycle of one age-group is equal in length to any lunar cycle, then this equality will not be present in any other age-group. Similarly, if the average for a whole population corresponds with a lunar cycle, the correspondence is unlikely to be present in another population having a different age-distribution. Since Arrhenius's average for the menstrual cycle was derived from data which mostly gave the time interval in whole weeks, while in ours the individual averages err by not more than $\pm 0 \cdot 1$ day, his conclusion can no longer be accepted.
An exhaustive examination of the number of our cases starting to menstruate on each day of each kind of lunar month has been made, involving statistical and graphical tests. No correlation of any kind was found. Arrhenius's data were known by him to be inaccurate, and they showed a systematic error, inherent in their mode of collection, which itself could give the appearance of a lunar periodicity. Our data are far more accurate and do not contain this source of error. We therefore conclude that there is no causal relation between the moon and menstruation, and that the well-known approximate coincidence in the duration of the cycles is fortuitous.

A full account of this investigation, embodying a number of other conclusions, will appear in October in the Journal of Obstetrics and Gynaecology of the British Empire.

University of Birmingham.

Donald L. GUNA.

University of Bristol.

59 Harley Street, W.1.

Penelope M. Jenkin.

Alistatr L. Guny.

${ }^{1}$ Fox, H. M., Proc. Roy. Soc., B, 95, 523-550 (1923).

${ }^{2}$ De Lce, J. B., "Principles and Practice of Obstetrics", p. 18 (6th edit., 1933. Philadelphia and London).

${ }^{3}$ Arrhenius, S., Skand. Arch. Physiol., 8, 36--416 (1898).

\section{Action of Radium Rays on the Growth of Cells in Vitro}

Numerous experiments on gamma irradiation of cells cultivated in vitro have demonstrated their high radio-resistance to doses designed to produce a lethal effect. Sub-lethal doses have the effect of producing changes in the mitotic activity of the culture, while the lethal dose has so far only been observed as a delayed effect.

In continuation of our earlier experiments ${ }^{1}$ we tried to work out a suitable dosage and method of application which would enable us to produce in the irradiated culture itself graded effects of radium from very slight inhibition of growth to death of the whole colony.

This was achieved in the following way. The source of rays consisted of a plate $1 \mathrm{sq}$. $\mathrm{cm}$. in area, containing $5.9 \mathrm{mgm}$. of radium element with a filter of $0.05 \mathrm{~mm}$. monel metal. With this filtration and with the use of a mica plate of $0.15 \mathrm{~mm}$. thickness for the culture, 12 per cent of the soft and 58 per cent of the hard $\beta$-rays were available, in addition to the $\alpha$-rays. By means of a simple centralizer, it was possible to conduct all the experiments under identical irradiation intensities. The radiations were applied to pure cultures of chick's mesenchyme cells cultivated in the hanging drop according to Carrel's method.

In contrast with most investigations hitherto published, irradiations were carried out in a refrigerator at $+6^{\circ} \mathrm{C}$. By this method of irradiation in the cold, we succeeded in investigating the various effects of radium treatment on the irradiated culture itself.

In all cases where more marked effects are to be produced by prolonged exposure, it is absolutely essential to suspend active cell life during the whole period of irradiation. With the usual method of treatment in the warm, the cells continue to migrate and divide during irradiation and the culture proceeds to develop; however, if the activity of a culture is suspended by cold, it accumulates the 